\title{
Automatic Summarization of Voicemail Messages Using Lexical and Prosodic Features
}

\author{
KONSTANTINOS KOUMPIS \\ Vienna Telecommunications Research Center \\ and \\ STEVE RENALS \\ University of Edinburgh
}

This aticle presents trainable methods for extracting principal content words from voicemail messages. The short text summaries generated are suitable for mobile messaging applications. The system uses a set of classifiers to identify the summary words with each word described by a vector of lexical and prosodic features. We use an ROC-based algorithm, Parcel, to select input features (and classifiers). We have performed a series of objective and subjective evaluations using unseen data from two different speech recognition systems as well as human transcriptions of voicemail speech.

Categories and Subject Descriptors: H.5.2 [Information Interfaces and Presentation]: User Interfaces-Natural language, Interaction styles, Evaluation/methodology; I.2.7 [Artificial Intelligence]: Natural Language Processing-Speech recognition and synthesis, Text analysis; H.1.2 [Models and Principles]: User/Machine Systems-Human factors, Human information processing; H.5.1 [Information Interfaces and Presentation]: Multimedia Information SystemsAudio input/output, Evaluation / methodology

General Terms: Languages

Additional Key Words and Phrases: Voicemail, automatic summarization, prosody, feature subset selection, receiver operating characteristic, short message service

\section{INTRODUCTION}

The increased proliferation of audio content has recently motivated several projects in the field of extracting and accessing information from audio archives.

This work was supported by EPSRC ROPA Award GR/R23954.

Authors' addresses: K. Koumpis, Vienna Telecommunications Research Center-ftw, Donau City Strasse 1, 1220 Vienna, Austria; email: koumpis@ftw.at; S. Renals, Centre for Speech Technology Research, University of Edinburgh, 2 Buccleuch Place, Edinburgh EH8 9LW, UK; email: s.renals@ed.ac.uk.

Permission to make digital or hard copies of part or all of this work for personal or classroom use is granted without fee provided that copies are not made or distributed for profit or direct commercial advantage and that copies show this notice on the first page or initial screen of a display along with the full citation. Copyrights for components of this work owned by others than ACM must be honored. Abstracting with credit is permitted. To copy otherwise, to republish, to post on servers, to redistribute to lists, or to use any component of this work in other works requires prior specific permission and/or a fee. Permissions may be requested from Publications Dept., ACM, Inc., 1515 Broadway, New York, NY 10036 USA, fax: +1 (212) 869-0481, or permissions@acm.org.

(C) 2005 ACM 1550-4875/05/0200-0001 $\$ 5.00$ 
Some notable successes have been Spoken Document Retrieval (SDR) and Named Entity (NE) extraction. A number of SDR systems operating on an archive of broadcast news were evaluated as part of the Text REtrieval Conference (TREC) from 1997-2000, producing the important result that retrieval performance on Automatic Speech Recognition (ASR) output was similar to that obtained using human-generated reference transcripts with little or no dependence on transcription errors [Garofolo et al. 2001]. This is not the case for all tasks which involve accessing information in spoken audio: it has been observed that the accuracy of NE identification is strongly correlated with the number of transcription errors [Kubala et al. 1998; Gotoh and Renals 2000; Palmer et al. 2000].

This article is about the generation of short text summaries of voicemail messages. Automatic summarization can be defined as the distillation of the most important information from a source, producing an abridged version, from a particular user and task [Mani and Maybury 1999]. The majority of research in this area has been concerned with the summarization of written text, reviewed by Mani [2001]. The growth of information and communication systems that deal with audio and visual media has stimulated the need to expand summarization systems from text to multimedia. For example, the existence of automatic speech summarizers would enable many practical applications such as the construction of automatically annotated audio archives, integrated mixed media communication systems and innovative multimodal interfaces.

A complete speech summarization system demands both spoken language understanding and language generation and is well beyond the current state-ofthe-art. However, it is possible to use simpler techniques to produce summaries that are of some use. The earliest reported work in speech summarization concerned the generation of crude summaries based on acoustic emphasis [Chen and Withgott 1992] and the classification of parts of dialogue [Rohlicek et al. 1992]. More recently, with the advent of large vocabulary speaker-independent continuous ASR, speech summarization research has focused on the application of text-based methods to ASR output [Valenza et al. 1999; Hori and Furui 2000; Zechner 2001]. At the same time, researchers have begun to combine prosodic, acoustic, and language information in an attempt to achieve results that are more robust than those of single sources. Application domains include identification of speech acts [Warnke et al. 1997], sentence and topic segmentation [Hirschberg and Nakatani 1998; Shriberg et al. 2000], and NE identification [Hakkani-Tür et al. 1999].

Voicemail involves a conversational interaction between a human and a machine with no feedback from the machine. Voicemail systems can record and store voice messages digitally while the user is away or simply unavailable and can be reviewed upon the user's return. Alternatively, the user can call in on a touchtone phone and review stored messages. Voicemail messages are typically short, conveying the reason for the call, the information that the caller requires from the voicemail recipient, and a return telephone number.

The slow, sequential nature of speech makes it hard to find important information quickly. Although several advances in voicemail retrieval schemes 


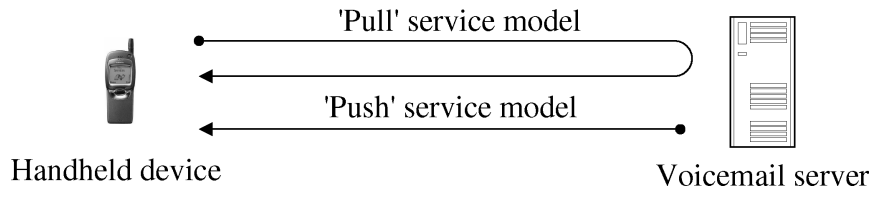

Fig. 1. Pull and push service models for accessing voicemail. The pull model employs a conventional request/response approach similar to that of the Web-a user enters a URL (the request) which is sent to a server, and the server answers by sending a Web page (the response). In the push model, content is delivered to the handheld device without a specific user request.

related to pause removal for faster playback and efficient audio coding have been proposed [Kato 1994; Paksoy et al. 1997], the limitations of the old paradigm remain. Users of voicemail systems, on the receipt of a notification, have to call their voicemail system, and download/listen to their actual/compressed messages. The ScanMail system [Hirschberg et al. 2001] allows users to browse and search the full message transcription of their voicemail messages by content through a graphical user interface. However, voicemail users are likely to want to receive their messages on handheld devices-especially for messages taken by voicemail systems other than the one provided by the network operator, for example, home or corporate voicemail system. In general, there is a lot of time-sensitive content in voicemail which the user cannot access either because it is not known when it becomes available (i.e., lack of notification mechanism), or because the notification refers only to changes in status (e.g., arrival of new messages) and not to actual content.

We have proposed an efficient voicemail retrieval scheme [Koumpis et al. 2001] which "pushes" text summaries of incoming messages to the handheld device directly from a server without an explicit user request. Figure $1 \mathrm{com}$ pares the two approaches for accessing voicemail content. In our architecture, the spoken messages collected by the voicemail system are forwarded to the content server where they are automatically transcribed and summarized. There is no restriction on the location of the voicemail system so access to answering services other than the one provided by the network operator is possible. The message initiator contacts the gateway over the Internet and delivers the messages. The gateway examines the message and performs the required encoding and transformation. The messages are then transmitted hop-by-hop in the mobile network to the mobile client. The message initiator is then notified by the gateway about the final outcome of the operation.

Automatically produced text summaries from voicemail messages may serve multiple goals such as the rapid digest of content, and the indexing of messages with the intention of retrieving the original recordings when more information is needed. Voicemail summarization has several features that differentiate it from conventional text summarization.

(1) Typical voicemail messages are short: the average duration of a voicemail message is $40 \mathrm{~s}$ in the work reported here.

(2) The summaries are extremely terse; in this case designed to fit into a 140 character text message, and therefore coherence and document flow (style) are less important than content. 
(3) Only one speaker speaks at a time, and due to the relatively short message length, segmentation is unnecessary (in contrast to spoken dialogues or broadcast news).

(4) Since the voicemail messages are transcribed by an ASR system, a significant word error rate (WER) must be assumed.

A number of techniques have been proposed to extract key pieces of information from voicemail messages. Huang et al. [2001] discuss three approaches to extract the identity and phone number of the caller: (1) 200 handcrafted rules; (2) grammatical inference of subsequential transducers; and (3) log-linear classifiers using a set of 10,000 bigram and trigram features. Jansche and Abney [2002] propose a phone number extractor based on a two-phase procedure that employs a handcrafted component derived from empirical data distributions, followed by a decision tree. These techniques rely explicitly on lexical information and the best-performing methods are based on handcrafted rules.

In this article, we present an approach to voicemail summarization based on the extraction of content words from the message transcription. Each word is characterized by a set of lexical and prosodic features, and we have trained classifiers on these feature vectors to discriminate summary words from nonsummary words. The set of features that we use for the classification was obtained using Parcel [Scott et al. 1998], an ROC-based feature selection methodology. We have carried out a number of experiments using a corpus of Voicemail speech, collected and transcribed by IBM [Padmanabhan et al. 1998], in which the behavior of our summarization approaches, utilizing speech recognizers with varying error rates, was evaluated utilizing both objective error measurements (with respect to a human-generated reference) and subjective user tests.

\section{SUMMARIZATION AS A CLASSIFICATION PROBLEM}

We have adopted a word-extractive approach to voicemail summarization [Koumpis et al. 2001] in which a summary is defined as a set of content words extracted from the original message transcription. Given a spoken message $\mathcal{S}$, the word-extractive summarization can be framed as the mapping of each transcribed word into a predefined summary class. This classification problem is hard since there can be a large degree of within-class variability relative to the between-class variability. Increasing the dimensionality of the feature space can enhance the training set discrimination but at a cost to generalization performance. If a gold standard reference is available with summary class labels for each word, then this approach can be evaluated using standard metrics based on the true positive and true negative rates, also known as sensitivity and specificity:

$$
\begin{aligned}
& \text { sensitivity }=\frac{\mathrm{TP}}{\mathrm{TP}+\mathrm{FN}}=\text { true positive rate, } \\
& \text { specificity }=\frac{\mathrm{TN}}{\mathrm{TN}+\mathrm{FP}}=\text { true negative rate, }
\end{aligned}
$$

where TP is the number of true positives (when a word is correctly classified as belonging to a class), $\mathrm{TN}$ is the number of true negatives, and $\mathrm{FP}$ and $\mathrm{FN}$ 


\begin{tabular}{|c|c|c|}
\hline \multicolumn{3}{|c|}{ Priority-based Categorization } \\
\hline Category & Training Set & Test $42 \& 50$ \\
\hline high & $37.4 \%$ & $29.3 \%$ \\
\hline medium & $51.4 \%$ & $54.3 \%$ \\
\hline low & $11.1 \%$ & $16.3 \%$ \\
\hline \multicolumn{3}{|c|}{ Content-based Categorization } \\
\hline Category & Training Set & Test $42 \& 50$ \\
\hline technical & $13.1 \%$ & $5.3 \%$ \\
\hline office & $16.9 \%$ & $23.4 \%$ \\
\hline business & $38.7 \%$ & $35.1 \%$ \\
\hline family & $5.9 \%$ & $12.8 \%$ \\
\hline friends & $16.4 \%$ & $12.8 \%$ \\
\hline private & $9.0 \%$ & $10.6 \%$ \\
\hline
\end{tabular}

are the numbers of false positives and false negatives, respectively. A receiver operating characteristic (ROC) curve gives a compound representation of sensitivity and specificity by plotting sensitivity against [1-specificity] [Zweig and Campbell 1993; Provost and Fawcett 2001]. For a binary classifier, the sensitivity and specificity are typically controlled by an acceptance threshold: for a strict threshold, the sensitivity will be low, while the specificity will be very high. If the threshold is lowered, specificity will fall, while sensitivity will rise. In this way, we can compare classifiers at particular operating points.

For a given task, two classifiers may be compared using their ROC curves. One classifier dominates another classifier if it has a higher sensitivity at all specificities; in other circumstances, one classifier may be more sensitive at some specificities and the other may be more sensitive at others (i.e., the curves cross). To obtain maximal sensitivity at all specificities, Provost and Fawcett [2001] showed that a set of component classifiers could be combined to give a composite classifier whose ROC curve is defined by the convex hull of the component classifier ROC curves. This convex hull is referred to as the maximum realizable ROC (MRROC) curve. Any operating point on the MRROC curve can be achieved by switching between the classifiers corresponding to the vertices of the convex hull.

\section{THE VOICEMAIL CORPUS}

We have used the IBM Voicemail Corpus-Part I [Padmanabhan et al. 1998], distributed by the Linguistic Data Consortium (LDC). This corpus contains 1801 messages (14.6 hours, averaging about 90 words per message). We used two test sets: the 42-message development test set distributed with the corpus (referred to as test42) and a second 50-message test set provided by IBM (test50). The messages in test 42 are rather short, averaging about 50 words per message, whereas the messages in test50 are closer to the training set average of 90 words per message. The messages in this corpus may be categorized as in Table I [Koumpis 2004]. 
We built a hybrid multilayer perceptron (MLP) / hidden Markov model (HMM) speech recognizer for the voicemail task [Koumpis and Renals 2000, 2001]. The essence of the hybrid approach is to train neural network classifiers to estimate the posterior probability of context-independent phone classes, then to use these probabilities (converted into likelihoods by dividing with the priors) as inputs to a HMM decoder [Morgan and Bourlard 1995]. The system used two MLPs one trained using perceptual-linear prediction acoustic features, the other using modulation-filtered spectrogram features. The log posterior probabilities estimated by the two networks were averaged to produce an overall log posterior probability estimate. During speech recognition training, we reserved the last 200 messages of the corpus as a development set, resulting in a 1601-message training set. An initial trigram language model was estimated using the training transcriptions. This training set was augmented with those sentences from the Hub-4 Broadcast News and Switchboard language model training corpora which had a low perplexity with respect to the initial language model and the language model reestimated. We used a pronunciation dictionary containing around 10,000 words derived from the training data with pronunciations obtained from the SPRACH broadcast news system [Robinson et al. 2002], plus 1,000 new words with pronunciations mainly constructed following the rules used to construct the broadcast news dictionary. The OOV rates were $1.6 \%$ on test 42 and $2.0 \%$ on test50. Additionally, we used 32 manuallydesigned compound words [Saon and Padmanabhan 2001]. The average test set WERs were $41.1 \%$ on test 42 and $43.8 \%$ on test50. We denote these transcriptions SR-SPRACH. Additionally, we obtained a second set of transcriptions (denoted SR-HTK) using the more complex HTK Switchboard system, adapted to the Voicemail corpus [Cordoba et al. 2002]. The WER for SR-HTK was 31\% for both test sets.

We annotated summary words in 1,000 messages of the Voicemail corpus. The first 800 messages were used as a summarization training set, and the last 200 were used as the development set. The transcriptions supplied with the Voicemail corpus include marking of NEs, and we built on this using the following scheme:

(1) pre-annotated NEs were marked as targets unless unmarked by later rules;

(2) the first occurrences of the names of the speaker and recipient were always marked as targets, while later repetitions were unmarked unless they resolved ambiguities;

(3) any words that explicitly determined the reason for calling, including important dates/times and action items were marked;

(4) words in a stopword list with 54 entries were unmarked;

All annotation was performed using the human transcription only (no audio).

As shown in Table II, the compression ratio in our training, development and testing material was in the range of $19 \%$ to $31 \%$. To assess the level of interannotator agreement, we compared the performance of sixteen human annotators who were asked to create word-extractive summaries for five messages at a compression ratio of $20-30 \%$. Fourteen out of sixteen of the annotators 
Table II. Voicemail Content Word Annotation

\begin{tabular}{l|c|c|c|c}
\hline & Training & Development & Test42 & Test50 \\
\hline Messages & 800 & 200 & 42 & 50 \\
Transcribed words & 66049 & 17676 & 1914 & 4223 \\
Total content words & 20555 & 5302 & 561 & 820 \\
$\quad$ Proper names & 2451 & 666 & 111 & 170 \\
Phone numbers & 3007 & 577 & 120 & 190 \\
Dates and times & 1862 & 518 & 46 & 81 \\
Other & 13235 & 3541 & 284 & 379 \\
Compression ratio & $31 \%$ & $30 \%$ & $29 \%$ & $19 \%$ \\
\hline
\end{tabular}

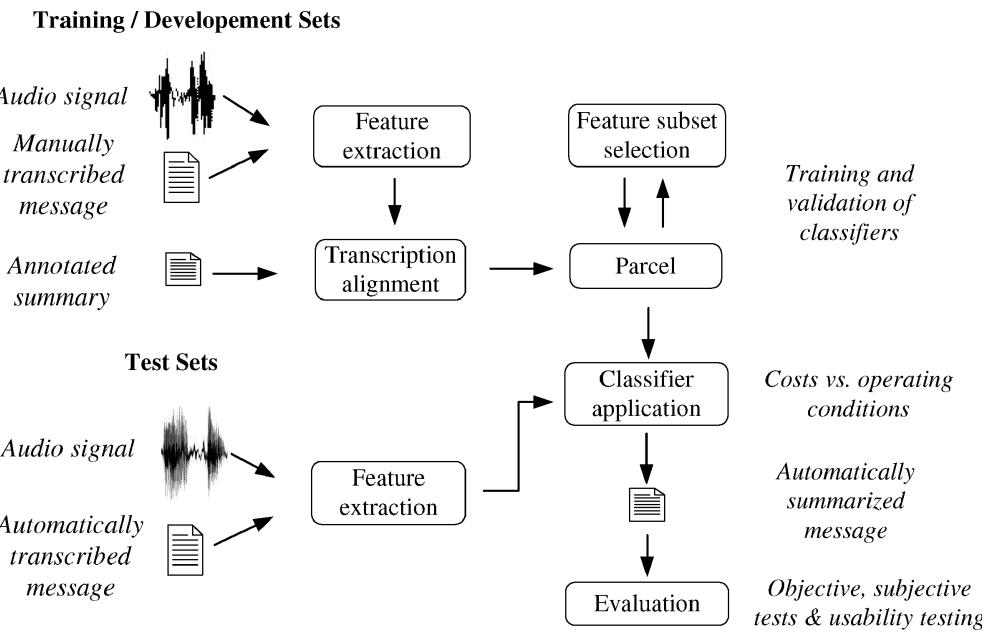

Fig. 2. An overview of the word-extractive summarization approach based on systematic comparisons and combination of patterns present in spoken audio.

produced their summaries by progressively eliminating irrelevant words (rather than selecting content words), and, in nearly all cases, the annotators tended to a compression ratio of $29-30 \%$. Interannotator agreement may be measured by the $\kappa$ statistic

$$
\kappa=\frac{\mathrm{P}_{\mathrm{o}}-\mathrm{P}_{\mathrm{e}}}{1-\mathrm{P}_{\mathrm{e}}}
$$

where $P_{o}$ is the proportion of times the annotators agree, and $P_{e}$ is the expected chance agreement. In this case, the average value of $P_{o}$ was 0.79 , resulting into a $\kappa$ of 0.48 , indicating a relatively good level of agreement.

\section{LEXICAL AND PROSODIC FEATURES}

The architecture of the voicemail summarization system is shown in Figure 2. Lexical information is obtained from the ASR transcriptions, while prosodic features are extracted from audio data using a signal processing algorithms or (in the case of pause and durational features) can be extracted by the speech recognizer. Each word in the transcription is represented by a set of lexical and prosodic features (listed in Table III). 


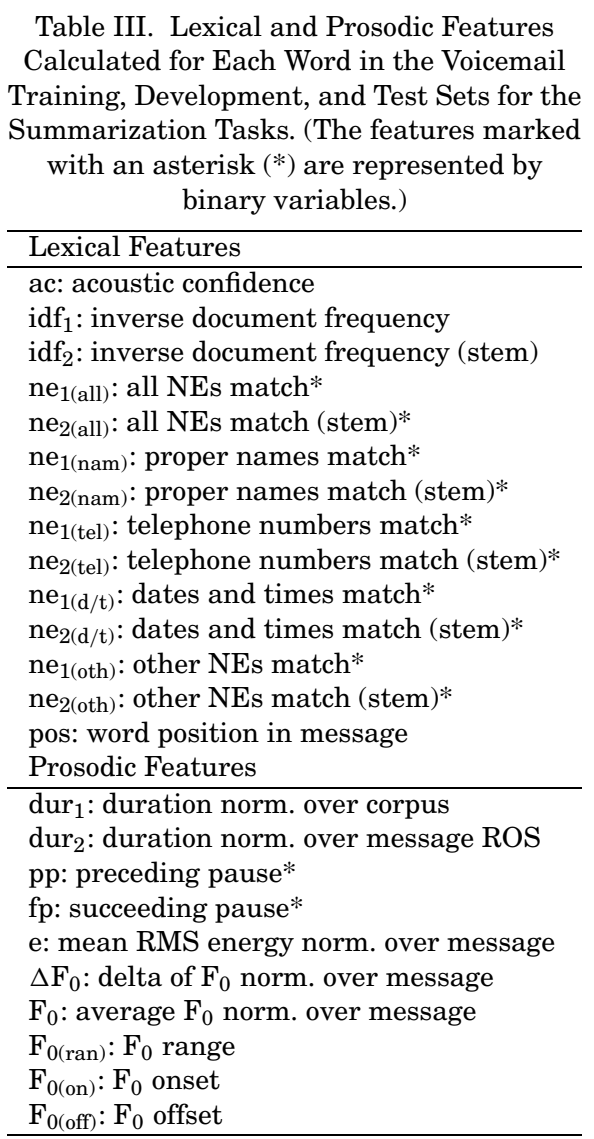

\subsection{Lexical Features}

For each word in the training, development, and test sets we calculated scores corresponding to IDF, NE matching, word positioning, and acoustic confidence. Another potentially useful feature not considered in our implementation is part of speech (POS).

4.1.1 Inverse Document Frequency. Inverse document frequency (IDF) [Robertson and Sparck Jones 1997] is an information retrieval measure that models the fact that words which occur only in a few messages are likely to be more informative than words which occur often in the entire corpus. For a term $\mathrm{w}_{\mathrm{i}}$, IDF is defined as

$$
\mathrm{IDF}_{\mathrm{w}_{\mathrm{i}}}=\log \frac{\mathrm{N}}{\mathrm{n}_{\mathrm{w}_{\mathrm{i}}}},
$$

where $\mathrm{N}$ is the number of messages in the training data and $\mathrm{n}_{\mathrm{w}_{\mathrm{i}}}$ is the number of messages that word $\mathrm{w}_{\mathrm{i}}$ occurs in. The $\mathrm{IDF}_{\mathrm{w}_{\mathrm{i}}}$ scores are then normalized by the number of terms in a message.

4.1.2 Named Entity Matching. Often the most important pieces of information in a message are the named entities: people, places, organizations, 
numbers, and dates. Identification of NEs in voicemail is less straightforward than for text. Rather than train or adapt a statistical NE identifier [Gotoh and Renals 2000] for voicemail, we used matches with an NE list of 3,400 entries, 2,800 of which were derived from the Hub-4 BN corpus [Stevenson and Gaizauskas 2000], the remainder derived from the Voicemail training data transcriptions.

4.1.3 Word Positioning. It is well known that the location of terms and sentences within a document can be a good indicator of their relevance to its content [Edmundson 1969]. We thus derived a related feature by associating each word in the voicemail transcriptions with a position index which was normalized across messages.

4.1.4 Acoustic Confidence. Ideally, we would like to extract only those words that were recognized correctly. Acoustic confidence measures, which may be extracted directly from the acoustic model for MLP/HMM speech recognizers [Williams and Renals 1999], quantify how well a recognized word matches the acoustic data, given the model.

\subsection{Prosodic Features}

Prosodic features concern the way in which sounds are acoustically realized and can disambiguate a text transcription (e.g., question or statement) or add new information (e.g., the speaker's emotional state). The main focus of existing computational theories of prosody is on stress and intonation, primarily as reflections of the lexical, syntactic, and information structures. One such theory developed by Pierrehumbert and colleagues [Pierrehumbert 1980; Beckman 1986] has three main distinguishing features. First, it assumes that phrasal intonation is comprised of a string of tones generated by a finite-state automaton. In general, this will consist of an optional boundary tone, a series of pitch accents, a phrase accent, and an optional final boundary tone. The second feature of the theory is the decomposition of the text to be associated with the tune into some metrical representation, indicating stressed and unstressed syllables. The third feature of the theory is the system of rules for associating tune with text. Thus, given some metrical representation of the text and intonational string of tones, there is a mechanism which associates the two. Ladd [1996] made another distinction for intonation between the contour interaction theories, which treat pitch accents on words as local differences of a global contour for the phrase, and the tonal sequence approaches, which treat phrasal tune as compositional from a sequence of elements associated with the word. Computational theories of prosody however have not yet progressed to a point where interesting generalizations can be made for an engineering approach to voicemail summarization. Hence, we decided to use raw prosodic features without addressing any formal theory of prosody in our modeling.

The manual annotation of prosody can be a very complex task, requiring a great deal of time and training. Most linguistic prosody research still relies heavily on the hand-labeling of speech, augmented by semi-automated computer analysis tools, since this is by far the most accurate way to obtain precise 
estimates of prosodic features. However, a machine learning approach to automatic speech summarization requires large quantities of data for training purposes for which prosody can not be expertly transcribed. Using signal processing algorithms or the output of the speech recognizer, we automatically extracted and computed the correlates of basic prosodic features associated with each transcribed word. These features can be broadly grouped as referring to pitch, energy, word duration, and pauses. Various versions for some features were used and a more detailed description of them follows.

4.2.1 Durational Features. The durations of the recognized words and phones may be extracted from the speech recognizer output (assuming that Viterbi decoding is used), and normalized within a message. Phone durations were expressed relative to the expected duration, normalizing to zero mean and unit variance. Word durations were normalized in a similar way with expected durations computed as a sum of the expected durations of constituent phones (using the pronunciation dictionary). We also extracted rate-of-speech (ROS) information using the enrate tool [Morgan et al. 1997] that calculates the syllable rate based on the computation of the first spectral moment of the low-frequency energy waveforms corresponding to a chosen time series segment.

4.2.2 Pause Features. Typically, pauses reflect the speaker's uncertainty in formulating utterances marking a conflict between speech planning and speech production. ASR systems in general treat silence as an additional subword unit and recognize it in the same way as other phone models. Therefore, from a practical perspective, pauses may be seen as the duration of the silence models which are easily extracted from the recognizer output. Due to the spontaneous nature of speech in Voicemail corpus, we decided not to use raw pause durations themselves. Instead we defined binary features for preceding and succeeding pause which took nonzero values if nonspeech regions preceding or succeeding a word exceeded a duration of $30 \mathrm{~ms}$. The selection of this threshold for the identification of pauses within a message was somewhat arbitrary and derived by studying a subset of forced alignments of the training data. Filled pauses which can be associated with the presence of important content words [Maclay and Osgood 1959; Shriberg 2001] were not treated in a special way during our analysis. Filled pauses were mapped to the same silence symbol as regular pauses.

4.2.3 $\mathrm{F}_{0}$ Features. The fundamental frequency $\left(\mathrm{F}_{0}\right)$, was computed using the pda function of the Edinburgh Speech Tools [Taylor et al. 1999]. This function implements a super-resolution pitch determination algorithm proposed by Medan et al. [1991]. To correct for estimation errors, we smoothed the output values using a 5-frame median filter.

We used a number of features derived from the estimate of $\mathrm{F}_{0}$ : the mean, range, and slope of the $F_{0}$ regression line over a window, ranging three frames preceding and following each word; the $\mathrm{F}_{0}$ onset (the first nonzero value in the segment); and the $\mathrm{F}_{0}$ offset (the last nonzero value in the segment). In case there were not enough $F_{0}$ samples in the examined window to calculate an adequate feature value (e.g., for short words such as articles), each missing 


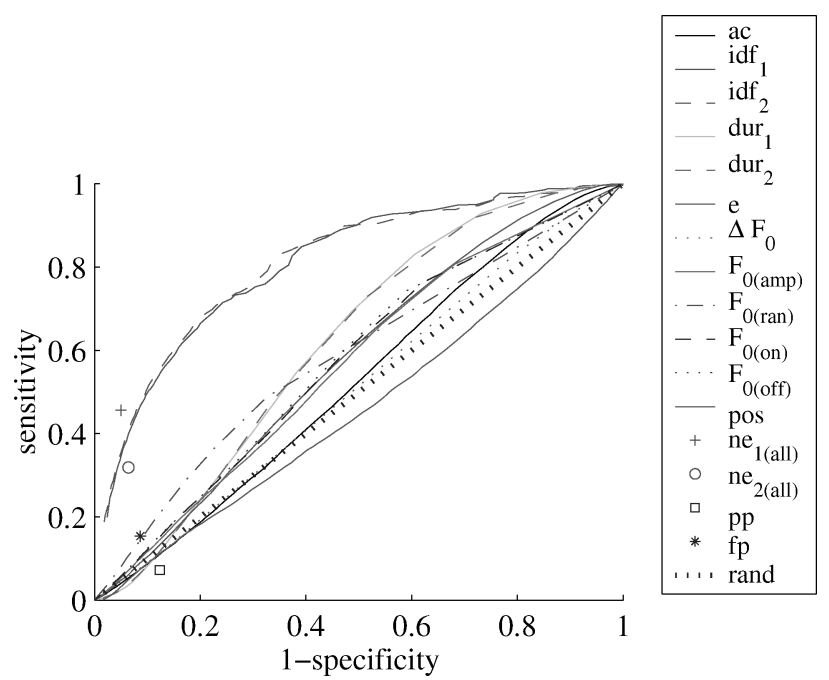

Fig. 3. The ROC curves produced by linear classifiers with respect to the development set for voicemail summarization, using the features listed in Table III (excluding those referring to classspecific NE matching).

value was set to the minimum available value from the words in the window's vicinity.

4.2.4 Energy Features. Energy features were calculated using the energy function of Edinburgh Speech Tools [Taylor et al. 1999]. This function calculates the RMS energy for each frame of the waveform.

\section{FEATURE SELECTION}

Each word in a transcribed voicemail message was represented by a vector of lexical and prosodic features as described. Some of these features provide more information for the task at hand than others, and some features may be redundant given other features. In this section, we assess the informativeness of these features for the voicemail summarization task first by considering single feature classifiers, then developing optimal feature subsets using an ROC-based algorithm, Parcel.

\subsection{Performance of Individual Features}

We investigated the informativeness of each of the lexical and prosodic features listed in Table III for the voicemail summarization task by training linear classifiers on each feature in turn.

5.1.1 Single Summary Class. In Figure 3, we show the ROC curves given by linear classifiers each trained on a single feature, testing on a development set. The best features for extracting summary words were lexical: IDF and $\mathrm{NE}$ matching. Of the prosodic features, the most important were durational, followed by energy. Features based on $\mathrm{F}_{0}$ information did not offer significant discrimination when used alone. 

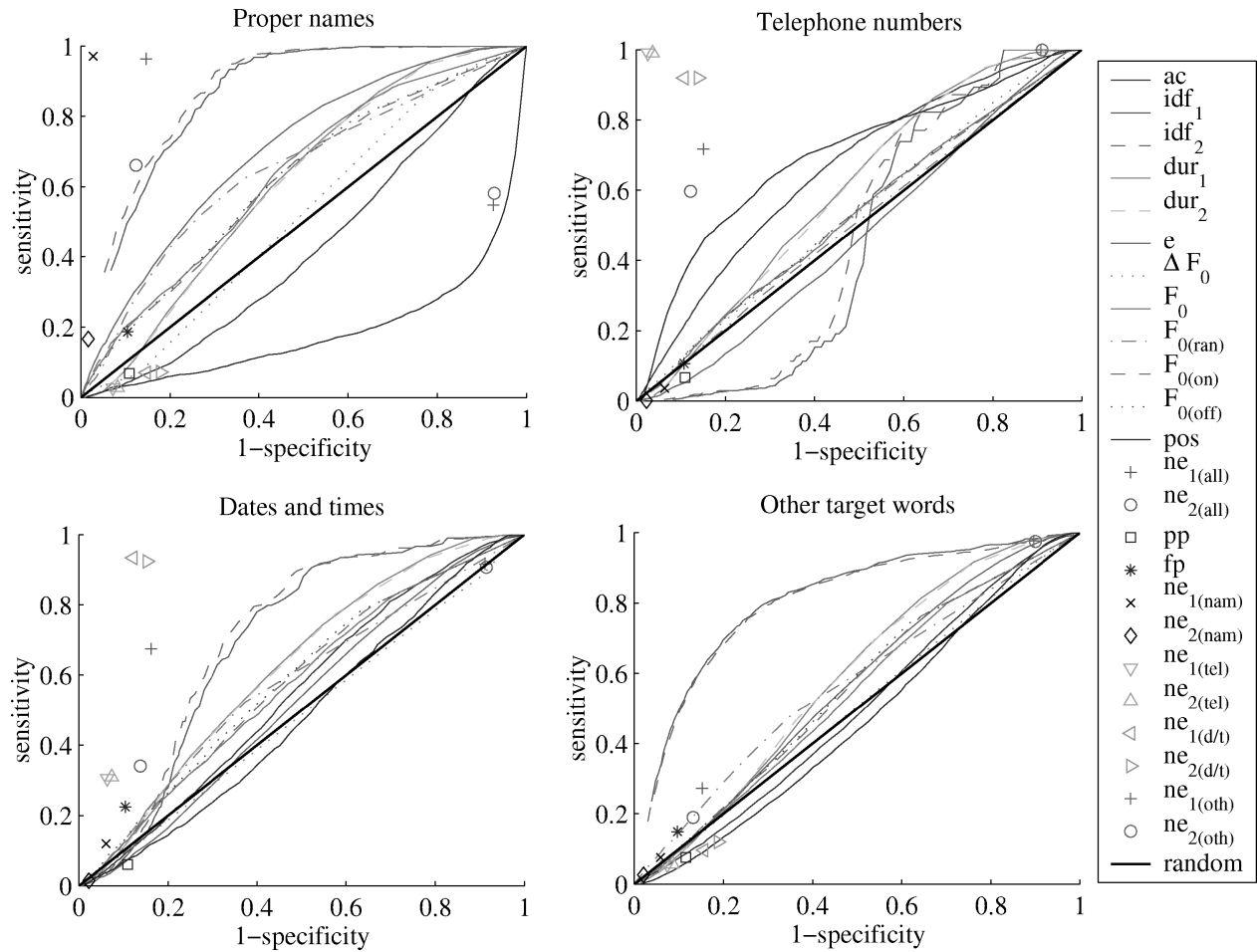

Fig. 4. The ROC curves produced by linear classifiers with respect to the development set for the four target classes using the individual features listed in Table III.

5.1.2 Separate Summary Classes. In Figure 4, we consider each of the summary classes (names, numbers, dates/times, other) separately and show an ROC curve for each feature and each summary class.

Proper names were identified very accurately by matching to named entity lists. In particular, matching with the unstemmed proper name list resulted in a very high true positive rate with a low false positive rate. The unstemmed general NE list also performed well, through stemmed variants were rather less accurate. IDF also offered good discrimination with the stemmed variant performing slightly but consistently better than the unstemmed variant $\left(\mathrm{idf}_{1}\right.$ ). Word position had strong negative correlation with this summary class, indicating that proper names are mostly positioned at the beginning of voicemail transcriptions where the position features have low values. Regarding the prosodic features, mean RMS energy, features based on $\mathrm{F}_{0}$, and duration (in descending order) gave useful discrimination. A weak correlation with following pauses was also observed.

Telephone numbers were also identified accurately by specific named entity lists. The date/time specific-named entity lists also matched well for this class (both name lists contain digits). Word position offered a good discrimination as telephone numbers typically appear towards the end of a message. IDF had an interesting correlation with this class. For words with low IDF, the correlation 
was strongly negative, while the correlation was slightly positive for words with an IDF above the average. It is also notable that the telephone numbers class had the highest acoustic confidence among all summary classes. Of the prosodic features, only the durational ones proved to be correlated with telephone numbers. The rest of prosodic features did not offer any useful discrimination.

The remaining two classes (dates/times and other) were less accurately identified by name matching. For dates and times, the specific named entity list was a good predictor as were the IDF features. The prosodic features were not particularly good predictors for this class; the best was following pause and the durational features. For the other class, matching to named entity lists was not useful; the most informative features were the IDF ones. Among the prosodic features the most useful were the word durations, energy and the $\mathrm{F}_{0}$ range.

\subsection{Selection of Multiple Features}

We used a feature selection approach in which the data was used to guide us to an optimal feature subset. Instead of demanding a single classifier and feature set (which would be optimized for a particular operating point in ROC space), we adopted an approach that maintained a set of classifiers and feature sets, enabling optimal performance at all points in ROC space. This approach, referred to as Parcel [Scott et al. 1998], builds on the notion of the MRROC curve formed as the convex hull of component ROC curves (Section 2).

Parcel is an iterative algorithm that selects those classifiers and feature sets that can extend the MRROC. It does not select a single feature subset (or classifier) but selects as many feature subset/classifier combinations that are required to maximize performance at all operating points. The operation of Parcel for feature selection is illustrated in Figure 5. In this example, the objective is to find a MRROC for a problem with a data set described by the features (a), (b), and (c). Sequential forward selection (SFS) is used in our implementation to search the feature space but any combinatorial search algorithm could be used instead. SFS starts with an empty set of features and, at each iteration, adds to the current subset the feature from those remaining that best satisfies the evaluation criterion.

Phase A. Estimate single feature classifiers and generate the ROC curves for each candidate feature. For continuous output classifiers vary a threshold over the output range to plot the ROC curve. The $\mathrm{MRROC}_{(\text {old }}$ is the diagonal.

Phase B. Form the convex hull of the ROC curves and retain those classifiers that correspond to the vertices of the convex hull. If $\mathrm{MRROC}_{(\text {new }}$ differs $^{1}$ from $\mathrm{MRROC}_{(\text {old })}$, the algorithm proceeds. Set $\mathrm{MRROC}_{(\text {old })}$ equal to $\mathrm{MRROC}_{\text {(new) }}$. In the example of Figure 5, as classifiers produce a continuous output to which different thresholds have been applied to predict class membership, the convex

\footnotetext{
${ }^{1}$ Each new classifier/feature either extends the existing convex hull or does not. The degree of difference is implementation dependent. In our experiments, we required a $5 \%$ minimum difference for the algorithm to proceed.
} 

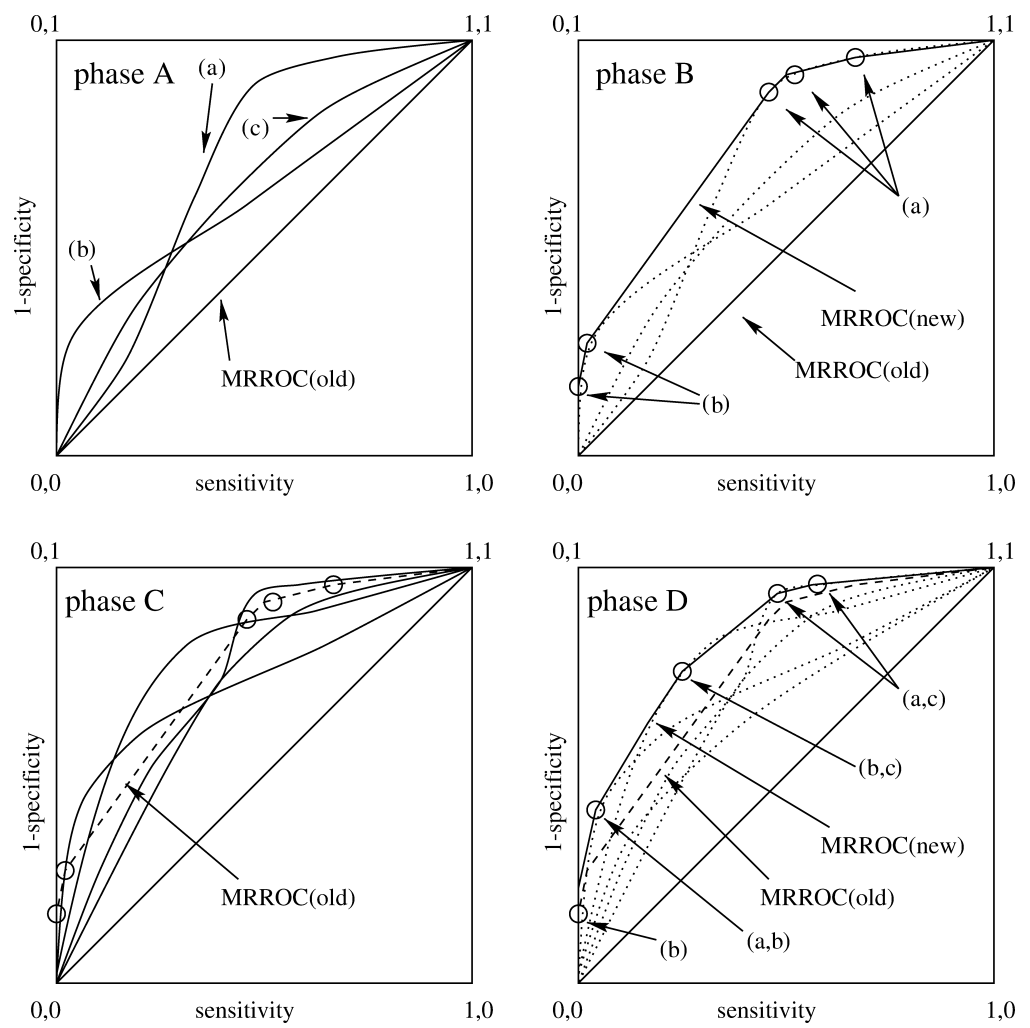

Fig. 5. The operation of the Parcel algorithm [Scott et al. 1998] in searching for the feature subsets that produce the MRROC. Only those systems whose operating points lie on the MRROC are saved as the rest can never be optimal. Clear visual comparisons and sensitivity analysis can be performed at each step of the algorithm's operation.

hull, MRROC $_{(\text {new) }}$, has five vertices. ${ }^{2}$ Two use feature subset (b), and three use (a).

Phase C. For each retained classifier $\mathrm{c}$ in the vertices of $\mathrm{MRROC}_{(\mathrm{old})}$, if there are $\mathrm{N}$ total features and $\mathrm{c}$ has $\mathrm{n}_{\mathrm{c}}$ features, then form $\mathrm{N}-\mathrm{n}_{\mathrm{c}}$ new classifiers each with $\mathrm{n}_{\mathrm{c}}+1$ features, formed by adding each remaining feature to the input feature set. Generate ROC curves for the new classifiers and recompute the convex hull.

Phase D. Retain those classifiers that are used to form the vertices of the convex hull (In Figure 5, two use feature subset (a, c), the others using (b), (a, b) and $(b, c))$. If the new convex hull, does extend the old convex hull, go to Phase C. Otherwise, terminate and return the set of classifiers that are the vertices of the convex hull.

Parcel requires no fixed classiciation algorithm to be used nor does it require a single classification algorithm to be used. It is possible to use multiple classification algorithms and to carry out the search for suitable classifiers to

${ }^{2}$ The convex hull of a set of points is the smallest convex hull that contains the points. 

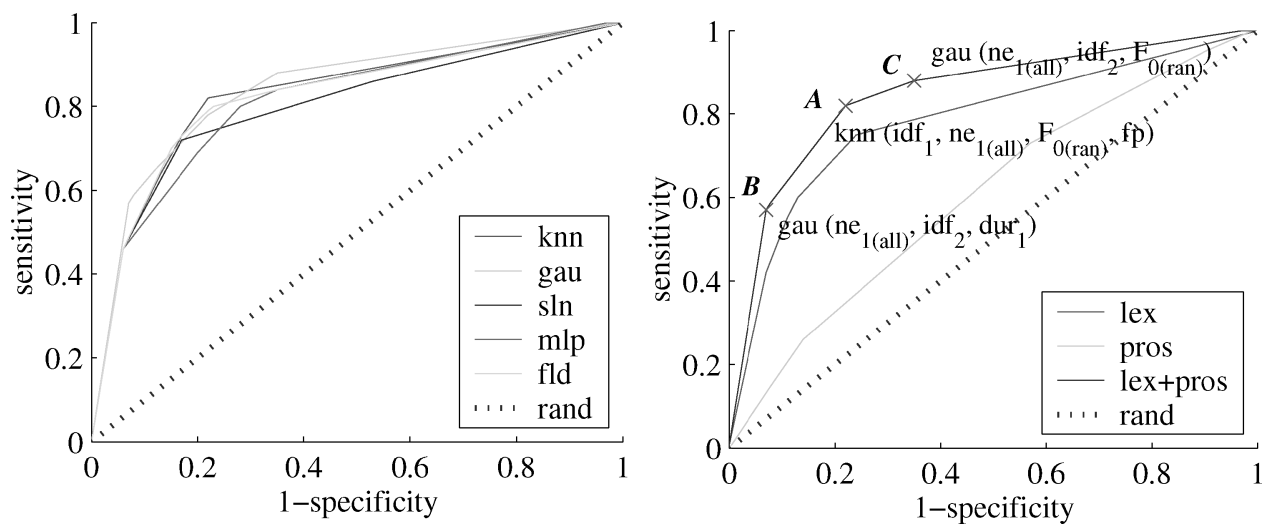

Fig. 6. The MRROC curves produced by Parcel on the development set, using the features listed in Table III (excluding those referring to class-specific NE matching). The left graph compares the role of the five classifiers employed, while the right one depicts the MRROC produced by all classifiers from lexical only, prosodic only, and lexical and prosodic features. Classifier A is optimal at moderate precision/recall trade-off; $\mathrm{B}$ is optimal at high precision; and $\mathrm{C}$ is optimal at high recall.

form the MRROC by not only varying the feature subset but also the classification algorithm. We employed five well-known classifiers within this framework: $\mathrm{k}$-nearest neighbor ( $\mathrm{knn}, \mathrm{k}=5$ ); Gaussian classifier (gau); single layer network ( $\ln )$; multilayer perceptron (mlp); and Fisher linear discriminant (fld). The selected classifiers follow different approaches resulting in different computational complexity. However, our preliminary experiments did not indicate any improvement in accuracy with the multilayer perceptron.

The training performance of the Parcel algorithm is shown in Figure 6 which graphs the MRROC curves of the development set for each of the classifiers (left), and selecting from lexical only, prosodic only, and all features (right). The classifiers, in this case, were trained on the human transcriptions. The k-nearest neighbors classifier gave very good trade-off between TP and FP for all four sizes of available training data. The Gaussian classifier produces relatively high numbers of both TP and FP covering a wide range of operating points. Finally, the results from the single layer network were relatively poor.

Although selecting from lexical features alone dominates selecting from prosodic features alone at all operating points, it can be seen that there is a clear benefit to augmenting the lexical features with prosodic features such as pitch range and pause information. We note that NE matching and IDF were the most important single features. Given a desired operating point in ROC space, Parcel enables us to choose a classifier that is optimal (with respect to the development set) for that point.

\section{EVALUATION}

The design of the automatic voicemail summarization system for mobile messaging requires trade-offs between the target summary length and the retaining of essential content words. The way message transcriptions are processed to construct summaries can affect everything from a user's perception of the 
service to the allocation and management of the mobile network's resources. Summaries are inherently hard to evaluate because their quality depends both on the intended use and on a number of other factors, such as how readable an individual finds a summary or what information an individual thinks should be included in it.

The following experiments were conducted using unseen test data and the questions we are looking to answer are the effects of speech recognition WER and of automatic summarization. Speech recognition WER was varied using human transcriptions (denoted SR-Human with 0\% WER) and the speech recognition transcriptions described in Section 3: SR-SPRACH (41-44\% WER) and SR-HTK (31\% WER). The effect of automatic summarization was obtained by comparing the automatic system described above with manual summarization and baseline automatic approaches (random selection of words and first $30 \%$ of the message). The latter baseline approach was based on the observation that important summarization content, such as the reason for calling and the caller's name, often appears at the beginning of messages rather than at the end. If the goal of summarization was to provide the caller's telephone number, then the end of a message might have provided a better alternative baseline.

\subsection{Objective Evaluation}

We have used the slot error rate (SER) [Makhoul et al. 1999] to compare an automatically-generated summary against a human-generated gold standard. The SER is analogous to the WER and treats substitution errors (correct classification, wrong transcription), insertion errors (false positives), and deletion errors (false negatives) equally. Of the classifiers forming the MRROC in the right of Figure 6, classifier A (using named entity match, IDF, $\mathrm{F}_{0}$ range, and following pause features) was used since it has the shortest Euclidean distance from the perfect classifier and is most appropriate if the aim is to minimize SER. Figure 7 shows these errors for summarization using classifier A applied to human (SR-Human), SR-SPRACH, and SR-HTK transcriptions for test42 and test50. Increasing speech recognition WER results in an increased SER. The highest WER system, based on SR-SPRACH, has a significantly higher deletion rate compared with SR-Human and SR-HTK which might occur because more summary words were misrecognized. Recognition errors also give rise to substitutions in the summaries (compared with the gold standard), and this can be seen by comparing the low level of substitutions for the system based on human transcriptions, compared with the systems based on SR-SPRACH and SR-HTK.

For SR-Human, $80 \%$ and $72 \%$ correct content and classification was achieved on test 42 and test50, respectively. For the SR-SPRACH, transcriptions, $49 \%$ and $47 \%$ correct classification was achieved on test 42 and test50, respectively. At the same time, for the SR-HTK, transcription scores were consistently higher, $60 \%$ and 55\% correct content and classification on test42 and test50, respectively. Deletion errors were $26 \%$ and $33 \%$ for SR-SPRACH, while for SR-HTK these were lower at $15 \%$ and $22 \%$. SER scores for test50 follow the same patterns as those for test 42 , though they are slightly poorer primarily due to a higher 

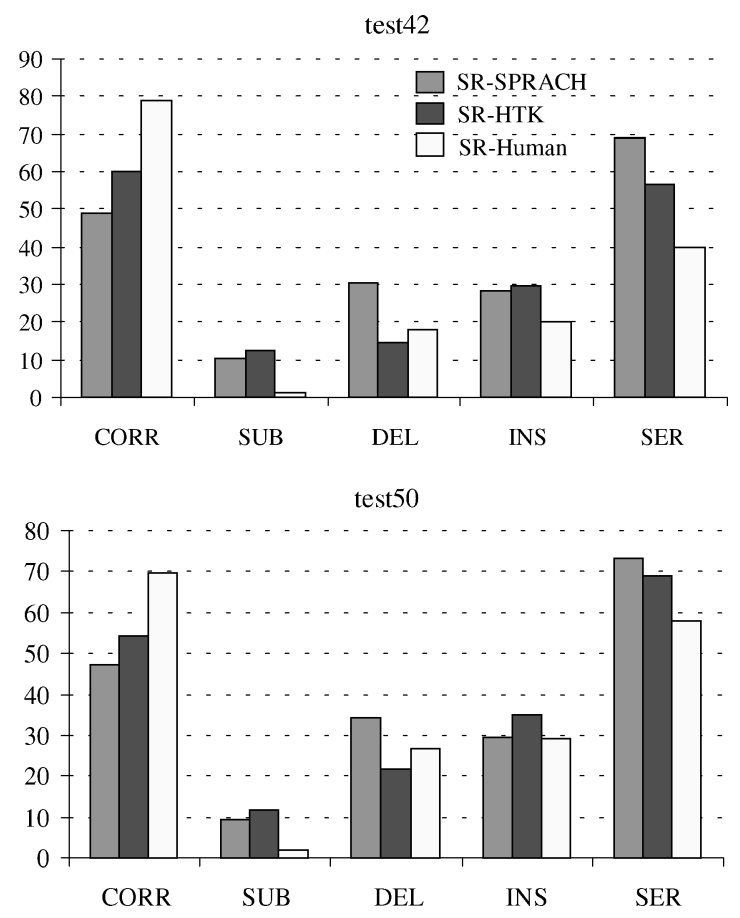

Fig. 7. Extractive summarization scores on test 42 and test50 for SR-SPRACH, SR-HTK, and SRHuman input, respectively.

deletions rate as a result of the relatively short gold standard summaries of the messages contained in the test50.

\subsection{Subjective and Usability Evaluation}

The quality of a service cannot be represented by a single measure but rather by a combination of several factors including learnability, effectiveness, and user satisfaction. Such factors must be assessed by having representative users interact with each application built. Usability testing ensures that application designs are on target and allow users to accomplish their tasks with ease and efficiency. Poor usability of voicemail summarization applications has a direct cost. Each time a user cannot determine the key content from a summary, they have to retrieve the original audio recording.

We have conducted some subjective and usability tests on the system in a controlled environment. These tests compared manual and automatic summaries presented in random order from SR-Human, SR-SPRACH, and SR-HTK transcriptions, along with the first $30 \%$ and a random (but sequentially ordered) set of the words in the human transcription. The mean opinion score (MOS) determined by 10 human subjects for 5 messages summarized in these 8 ways are shown in Figure 8. We found that subjects tended to agree more on which summaries are of low quality rather than high quality, and the overall $\kappa$ statistic was in the range 0.26 to 0.41 . The scores indicate that the automatic summaries are considered to be better than selecting the first $30 \%$ of words or random 


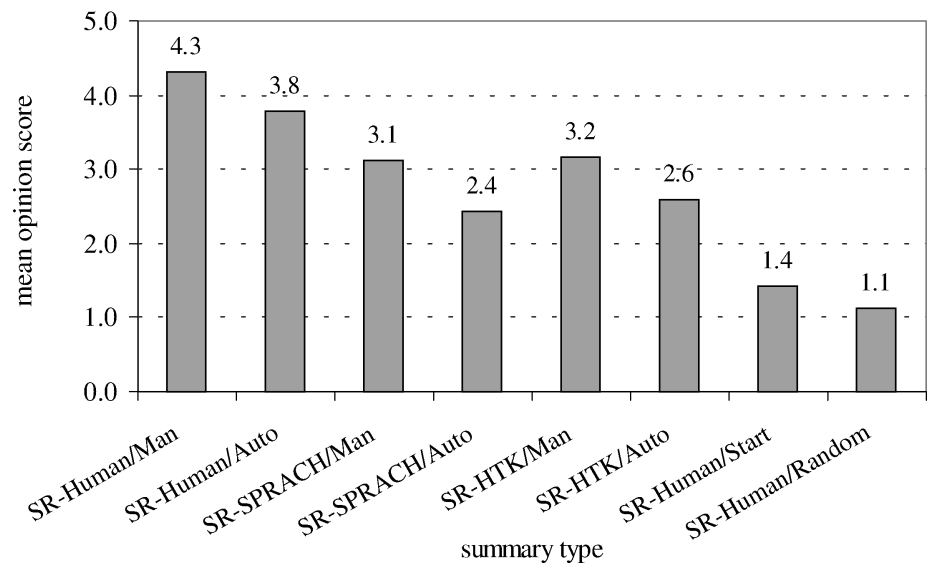

Fig. 8. Average MOS on 8 summaries for 5 messages from test 42 judged by 10 subjects.

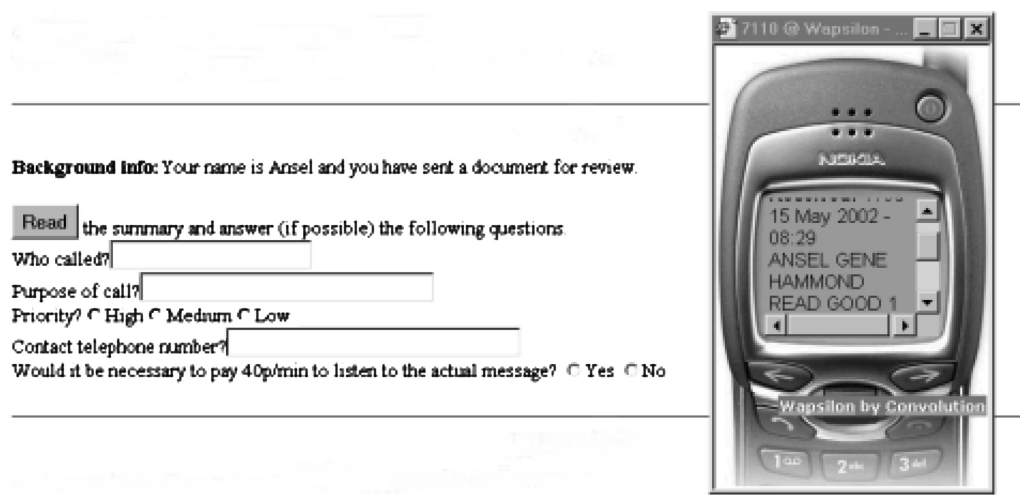

Fig. 9. Audiovisual interface used for summarization assessment allowing users to access the original audio and the text summaries.

selection but are inferior to the corresponding human-generated summaries. Moving from human to automatic summaries reduces the MOS by about 0.6 , whereas moving from a human transcription to a speech recognizer with 30 40\% WER reduces the MOS by over 1 point.

A second set of tests aimed to assess the summary quality in terms of comprehension. Subjects answered questions about message content (caller name?, reason for calling?, message priority?, contact number?) based on the audio and the text summaries. We used a WAP phone emulator to simulate transmitted summaries, and the audiovisual interface is shown in Figure 9. The tests were carried out by 16 subjects who were presented with the summaries and audio of 15 voicemail messages. The summaries used in the human and SRSPRACH transcriptions and the results are shown in Table IV. Human transcription was considerably more reliable in determining caller identity (94\% vs. 57\%), but there was less difference in determining the contact phone number $(82 \%$ vs. $80 \%)$. The users were able to determine the reason for calling with equal accuracy (78\%) for both types of transcriptions. The results indicate 
Table IV. Average Percentage of Correct Answers in Message Comprehension

\begin{tabular}{l|c|c}
\hline Question & SR-Human & SR-SPRACH \\
\hline caller name & $94 \%$ & $57 \%$ \\
reason for calling & $78 \%$ & $78 \%$ \\
priority & $63 \%$ & $58 \%$ \\
contact number & $82 \%$ & $80 \%$ \\
retrieve audio & $30 \%$ & $53 \%$ \\
\hline
\end{tabular}

Table V.

\begin{tabular}{l|c|c|r|l|r|c|c}
\hline \multicolumn{3}{c}{ SR-Human } & \multicolumn{4}{c}{ SR-SPRACH } \\
\hline & Low & Medium & High & & Low & Medium & High \\
Low & 7.0 & 8.6 & 0.0 & Low & 12.5 & 12.5 & 2.7 \\
Medium & 6.3 & 22.7 & 10.9 & Medium & 5.4 & 31.3 & 10.7 \\
High & 2.3 & 9.4 & 32.8 & High & 1.8 & 8.9 & 14.3 \\
\hline
\end{tabular}

Message priority confusion matrices as determined by 16 subjects who were initially presented with the automatically produced text summaries and later with the oiginal audio of 15 messages. Each column corresponds to the percentage of messages classified as low/medium/high priority based on text summaries and each row gives the percentage of messages classified as low/medium/high priority based on the original audio. The degree of consistency across judgements can be determined by summing up the diagonal values.

that summaries produced using automatic transcriptions are particularly useful for tasks such as determining the reason for calling, priority of messages, and contact numbers. It seems that users were able to associate the words included in summaries to make global judgements about the message content. This supports the hypothesis that even a few relevant words extracted from a transcription can lead to good message perception and allow potential action to be taken. The evaluation also showed that the users were much more likely to request the message audio when presented with summaries generated from the speech-recognized message, compared with summaries generated from human transcriptions ( $53 \%$ vs. $30 \%)$.

Message priority could be determined relatively accurately from the summaries (Table V): classifying priority as high/medium/low, the priority obtained from the summary agreed with that obtained from the audio $58 \%$ of the time for SR-SPRACH and $63 \%$ of the time for human transcriptions. The cases where the subjects completely misjudged the message priority from the text summaries were $2 \%$ (judged as high, while from the summary, they thought it was low) and 5\% (judged as low, while from the summary, they thought it was high). The above results suggest that transcription errors affect mainly the identity of the caller though they lead to $23 \%$ more retrievals of audio recordings as users were not confident that the information they read in a summary corresponded to the full and correct content of voicemail messages.

Figure 10 summarizes the time taken by users to answer the comprehension questions about the voicemail messages, comparing summaries based on human and SR-SPRACH transcriptions and the original audio. Although not directly comparable (since each message was used in one form only), the average comprehension time for speech recognition summaries was about $30 \%$ greater 


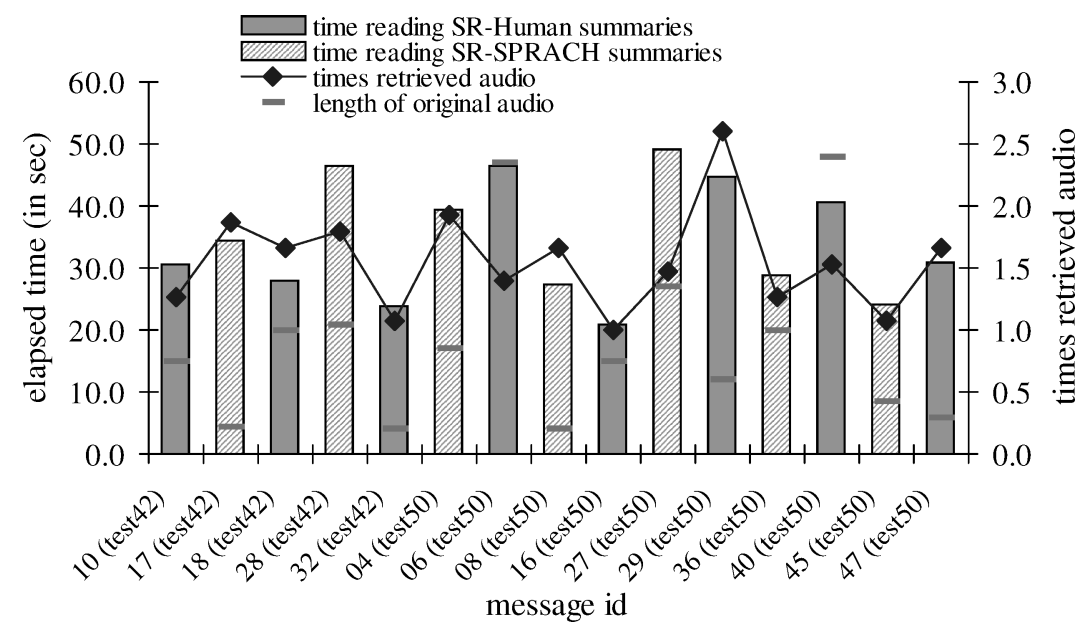

Fig. 10. Message comprehension times comparing accessing the original audio to summaries produced from human and SR-SPRACH transcripts.

than for the human transcription case. These times are about 1.5 times longer than performing the same task using the audio. Note that these figures include the time required to type the answers in the appropriate template fields (Figure 9). This favours the audio retrieval scenario where users can listen to the recording while typing their answers. At the same time, while retrieving the text summaries, they had to browse the mobile display to find the appropriate bit of information prior to typing it. In practice, retrieving the audio would also involve connection overheads such as typing a PIN. Despite the fact that in the experiment the digestion of text summaries was not found to be as rapid as that achieved by listening to the audio, the advantages of summarization, for example, indexing and uninterrupted information flow in noisy places need to be considered.

Finally, 13 out of the 16 subjects ( $81 \%$ ) who took part in this evaluation would likely use such a service regularly to access their voicemail messages while away from the office or home. This suggests that even average quality automatic summaries might be preferable given the elaborate nature of accessing spoken audio.

\subsection{Discussion}

Engineering-oriented metrics and user input can be correlated with system properties to identify what components of the system affect usability and to predict how user satisfaction will change when other trade-offs are made [Walker et al. 1998]. This evaluation framework was extended in Koumpis [2002] where the aim was to determine which metrics maximize summary quality and minimize delivery costs within this automatic voicemail summarization system for mobile messaging. One disadvantage of this framework is the amount of data required from subjective evaluations. Instead of solving for weights on the success and cost measures using multivariate linear regression as in Walker 
et al. [1998], one could use Parcel to calculate the role of each metric to the overall system performance. This is a straightforward and possibly much more robust process as the metrics are numerical values that can be used as inputs to simple classifiers that will be trained and validated using task completion as perceived by human subjects as an external criterion.

Although treating transcribed words independently proved to work relatively well and allowed us to study the correlation between word classes and a variety of features, it is expected that if modeling is extended beyond the word level, classification can be based on the expectations from syntax, semantics, and pragmatics and lead to better text coherence. HMMs are a well-developed probabilistic tool for modeling sequences of observations, although the amount of annotated data requirements will need to be addressed.

The effects of different message categories in the summarization task were not addressed in our experiments. It would be interesting, for instance, to find out what the performance would be after training the models using business messages and testing them on private messages. Although, some of our recent work [Koumpis 2004] examined the applicability of stochastic language models to the task of automatic voicemail categorization, it did not examine it in connection with the summarization approach presented herein.

\section{CONCLUSION}

In this article, we have presented a framework for voicemail summarization, based on the extraction of words from speech recognition transcriptions. The word extraction process operated by training classifiers to identify words as summary words or not, with each word represented by a vector of lexical and prosodic features. The features used in the summarizer were selected using Parcel, a method based on ROC curves, which returned a collection of feature sets and classifiers that together were optimal at all points in ROC space. Although lexical features (NE list matching and IDF) were most informative, we found that a significant improvement could be observed by augmenting them with some prosodic features.

We evaluated the resultant voicemail summarization system through comparison with human-generated gold standard summaries (using slot error rate) and through subjective user testing. We assessed the effect of transcription word error rate, comparing the performance of automatic summarization approaches with respect to transcriptions produced by hand and produced by recognizers with average word error rates of $31 \%$ and $42 \%$. The summarization slot error rate was dependent on the word error rate, but the difference between the two speech recognition systems was small; however, the human transcribed system was significantly better. We conducted a set of usability tests, using human subjects, based on mean opinion score of summaries and on a set of comprehension tests. The main results from these experiments were that the automatic summaries were inferior to human summaries, but there was a greater perceived quality difference between summaries derived from handand automatically-transcribed messages than between manual and automatic summarization. 


\section{ACKNOWLEDGMENTS}

We thank Mark Gales for providing us with the HTK transcriptions of the voicemail test sets and Mark Stevenson for providing the BN corpus derived NE lists. We also acknowledge discussions with Mahesan Niranjan and Rob Gaizauskas.

\section{REFERENCES}

Beckman, M. 1986. Stress and Non-Stress Accent. Foris Publications, Dordrecht, Holland/Riverton.

Chen, F. And Withgotт, M. 1992. The use of emphasis to automatically summarize a spoken discourse. In Proceedings of IEEE International Conference on Acoustics, Speech, and Signal Processing (ICASSP'92). San Francisco, CA. 229-232.

Cordoba, R., Woodland, P. C., ANd Gales, M. J. F. 2002. Improving cross task performance using MMI training. In Proceedings of IEEE International Conference on Acoustics, Speech, and Signal Processing (ICASSP'02). Orlando, FL. 85-88.

Edmundson, H. P. 1969. New methods in automatic extracting. J. ACM 16, 2, 264-285.

GAROFolo, J., LARD, J., AND VoorheEs, E. 2001. TREC-9 spoken document retrieval track: overview and results. In Proceedings of the 9th Text Retrieval Conference (TREC-9). Gaithersburg, MD.

Gotoh, Y. AND Renals, S. 2000. Information extraction from broadcast news. Philosophical Trans. Royal Soc. London, Series A, 358, 1295-1310.

Hakkani-Tür, D., Tür, G., StolCKe, A., AND Shriberg, E. 1999. Combining words and prosody for information extraction from speech. In Proceedings of Eurospeech. Budapest, Hungary. 19911994.

Hirschberg, J., Bacchiani, M., Hindle, D., Isenhour, P., Rosenberg, A., Stark, L., Stead, L., WhitTAKer, S., AND ZAMChick, G. 2001. SCANMail: Browsing and searching speech data by content. In Proceedings of Eurospeech. Aalborg, Denmark.

Hirschberg, J. AND NAKATANI, C. 1998. Acoustic indicators of topic segmentation. In Proceedings of International Conference on Spoken Language Processing (ICSLP'98). Sydney, Australia. 12551258.

HoRI, C. AND FurUI, S. 2000. Improvements in automatic speech summarization and evaluation methods. In Proceedings of International Conference on Spoken Language Processing (ICSLP'O0). Beijing, China. 326-329.

Huang, J., Zweig, G., AND Padmanabhan, M. 2001. Information extraction from voicemail. In 39th Annual Meeting of the Association for Computational Linguistics. Toulouse, France.

Jansche, M. AND ABNEY, S. 2002. Information extraction from voicemail transcripts. In Proceedings of the Conference on Empirical Methods in NLP. Philadelphia, PA.

KATO, Y. 1994. Voice message summary for voice services. In International Symposium on Speech, Image Processing and Neural Networks. Hong-Kong. 622-625.

KoumPIS, K. 2002. Automatic voicemail summarisation for mobile messaging. Ph.D. thesis, University of Sheffield, UK.

KoumPIS, K. 2004. Automatic categorization of voicemail transcripts using stochastic language models. In Proceedings of the 7th International Conference on Text, Speech and Language. Brno, Czech Republic. Lecture Notes in Computer Science.

Koumpis, K., LADAS, C., AND RENALS, S. 2001. An advanced integrated architecture for wireless voicemail retrieval. In Proceedings of the 15th IEEE International Conference on Information Networking. Beppu, Japan. 403-410.

Koumpis, K. And Renals, S. 2000. Transcription and summarization of voicemail speech. In Proceedings of International Conference on Spoken Language Processing (ICSLP'00). Beijing, China. 688-691.

Koumpis, K. And Renals, S. 2001. The role of prosody in a voicemail summarization system. In Proceedings of the ISCA Workshop on Prosody in Speech Recognition and Understanding. Red Bank, NJ. 87-92.

Koumpis, K., RENALS, S., AND Niranjan, M. 2001. Extractive summarization of voicemail using lexical and prosodic feature subset selection. In Proceedings of Eurospeech. Aalborg, Denmark. $2377-2380$.

ACM Transactions on Speech and Language Processing, Vol. 2, No. 1, February 2005. 
Kubala, F., Schwartz, R., Stone, R., and Weischedel, R. 1998. Named entity extraction from speech. In Proceedings of DARPA Broadcast News Transcription and Understanding Workshop. Lansdowne, VA.

LADD, D. R. 1996. Intonational Phonology. Cambridge University Press, Cambridge, UK.

Maclay, H. ANd OsGoOd, C. 1959. Hesitation phenomena in spontaneous english speech. Word 1, $19-44$.

Makhoul, J., Kubala, F., Schwartz, R., and Weischedel, R. 1999. Performance measures for information extraction. In Proceedings of the DARPA Broadcast News Workshop. Herndon, VA. 249-252.

Mani, I. 2001. Automatic Summarization. John Benjamins Publishing, Amsterdam, The Netherlands.

Mani, I. ANd Maybury, M. 1999. Advances in Automatic Text Summarization. MIT Press, Cambridge, MA.

Medan, Y., Yair, E., ANd ChaZAn, D. 1991. Super resolution pitch determination of speech signal. IEEE Trans. Acoustics, Speech Signal Process. 39, 1, 40-48.

Morgan, N. AND Bourlard, H. 1995. An introduction to hybrid HMM/connectionist continuous speech recognition. IEEE Signal Process. Mag., 25-42.

Morgan, N., Fosler, E., AND Mirghafori, N. 1997. Speech recognition using on-line estimation of speaking rate. In Proceedings of Eurospeech. Rhodes, Greece. 2079-2082.

Padmanabhan, M., Eide, E., Ramabhardan, G., Ramaswany, G., and Bahl, L. 1998. Speech recognition performance on a voicemail transcription task. In Proceedings of IEEE International Conference on Acoustics, Speech, and Signal Processing (ICASSP'98). Seattle, WA. 913-916.

Paksoy, E., McCree, A., Viswananthan, V., And Linn, J. 1997. A variable-rate CELP coder for fast remote voicemail retrieval using a notebook computer. In Proceedings of the IEEE Workshop on Multimedia Signal Processing. Princeton, NJ. 119-124.

Palmer, D., Ostendorf, M., And Burger, J. D. 2000. Robust information extraction from automatically generated speech transcriptions. Speech Comm. 32, 1-2, 95-109.

Pierrehumbert, J. 1980. The phonology and phonetics of english intonation. Ph.D. thesis, MIT, Cambridge, MA.

Provost, F. AND FAWCETt, T. 2001. Robust classification for imprecise environments. Machine Learn. 42, 3, 203-231.

Robertson, S. E. ANd Sparck Jones, K. 1997. Simple proven approaches to text retrieval. Tech. rep., TR-356, Cambridge University Computer Laboratory, Cambridge, UK.

Robinson, A. J., Cook, G. D., Ellis, D. P. W., Fosler-Lussier, E., Renals, S. J., And Williams, D. A. G. 2002. Connectionist speech recognition of broadcast news. Speech Comm. 37, 27-45.

Rohlicek, J. R., Ayuso, D., Bates, M., Bobrow, R., Boulanger, A., Gish, H., Jeanrenaud, P., Meteer, M., AND Siu, M. 1992. Gisting conversational speech. In Proceedings of IEEE International Conference Acoustics, Speech, and Signal Processing (ICASSP'92). San Francisco, CA. 113-117.

Saon, G. and Padmanabhan, M. 2001. Data-driven approach to designing compound words for continuous speech recognition. IEEE Trans. Speech Audio Process. 9, 4, 327-332.

Scott, M., Niranjan, M., and Prager, R. 1998. Parcel: Feature subset selection in variable cost domains. Tech. rep., CUED TR-323, ftp://svr-ftp.eng.cam.ac.uk/pub/reports. Cambridge, UK.

ShriberG, E. 2001. To "errrr" is human: Ecology and acoustics of speech disfluencies. J. Int. Phonetic Ass. 31, 1, 153-169.

Shriberg, E., Stolcke, A., Hakkani-Tür, D., ANd Tür, G. 2000. Prosody-based automatic segmentation of speech into sentences and topics. Speech Comm. 32, 1-2, 127-154.

Stevenson, M. And Gaizauskas, R. 2000. Using corpus-derived named lists for named entity recognition. In Proceedings of Applied NLP and the North American Chapter of the ACL. Seattle, WA. 290-295.

Taylor, P., Caley, R., Black, A. W., and King, S. $1999 . \quad$ Edinburgh speech tools library. Tech. rep., ftp://ftp.cstr.ed.ac.uk. Edinburgh, UK.

Valenza, R., Robinson, T., Hickey, M., AND Tucker, R. 1999. Summarization of spoken audio through information extraction. In Proceedings of ESCA Workshop on Accessing Information in Spoken Audio. Cambridge, UK. 111-116.

Walker, M. A., Litman, D. J., Kamm, C. A., ANd Abella, A. 1998. Evaluating spoken dialogue agents with PARADISE: Two case studies. Comput. Speech Lang. 12, 3, 317-347. 
Warnke, V., Kompe, R., Niemann, H., AND Nöth, E. 1997. Integrated dialog act segmentation and classification using prosodic features and language models. In Proceedings of Eurospeech. Rhodes, Greece. 207-210.

Williams, G. And Renals, S. 1999. Confidence measures from local posterior probability estimates. Comput. Speech Lang. 13, 395-411.

ZECHNER, K. 2001. Automatic generation of concise summaries of spoken dialogues in restricted domains. In Proceedings of ACM SIGIR. New Orleans, LA. 199-207.

Zweig, M. H. AND CAMPBELL, G. 1993. Receiver-operative characteristic (ROC) plots: A fundamental evaluation tool in clinical medicine. Clinical Chem. 39, 561-577.

Received December 2003; revised February 2005; accepted April 2005 by Alex Waibel 